\title{
High frequency oscillations as a correlate of visual perception
}

\author{
Jasna Martinovic ${ }^{1} \&$ Niko A. Busch ${ }^{2,3}$
}

1- School of Psychology, University of Aberdeen, UK

2- Berlin School of Mind and Brain, Humboldt-Universität Berlin, Germany

3- Institute of Medical Psychology, Charité Universitätsmedizin Berlin, Germany

Pages: 20; Figures:0; Tables:0

Corresponding author:

Dr Jasna Martinovic

School of Psychology

University of Aberdeen

William Guild Building

Aberdeen

AB24 2UB

tel: +441224272240

fax: +44 1224273426

email: j.martinovic@abdn.ac.uk 


\begin{abstract}
Cortical oscillatory activity in the gamma-band range $(>30 \mathrm{~Hz})$ is a fundamental mechanism of neural coding that arises during a range of cognitive processes in both animals and humans. Since the first report on high frequency oscillatory synchrony between V1 neurons belonging to the same orientation column (Gray and Singer, 1989, PNAS, 86, 1698-1702), the role of such oscillations in visual perception has been extensively researched. Visual stimuli elicit an early, evoked gamma-band response and a later, induced (neither time nor phase-locked) response. An abundance of experimental evidence now links both evoked and induced high frequency oscillations to a range of visual stimulus properties. On the basis of early studies into gamma-band oscillations in vision, induced high frequency oscillatory activity has been proposed as a putative cortical mechanism of coherent percept formation and object representation while evoked high frequency activity was related to the processing of image features. Recent studies demonstrate that both evoked and induced gamma-band activity are correlated with the speed and accuracy of visual detection and discrimination. Furthermore, induced gamma-band oscillations in the visual cortex are also correlated with fixational eye movement patterns. These direct relations between gamma-band activity and the efficacy of visual perception strongly suggest that cortical high frequency synchronisations constitute a neural mechanism that subserves processes essential for the organised intake and analysis of visual information.
\end{abstract}

Keywords: gamma-band activity, EEG, high frequency oscillations, visual perception, object representation, neural coding, electrophysiology 


\section{Introduction}

\section{Dynamics of visual processing}

Perception is fundamentally a cognitive activity, a process of acquiring knowledge about

environmental objects and events for the purpose of appropriate action (Palmer, 1999). In order to guide behaviour aptly, perception has to effectively deal with large amounts of highly varied information. This is particularly true for visual perception. The complexity of the visual environment introduces many challenges: scenes need to be segmented and objects layered in space, a process that requires concurrent bottom-up and top-down contributions (Ullman, 2007). In this context, bottom-up contributions derive from processes that analyse incoming visual information with little recourse to stored knowledge, while top-down contributions arise from prior visual knowledge and the task set. Bottom-up segmentation of natural scenes cannot in itself lead to speedy and efficient processing, since commonly-present image ambiguities necessitate top-down inputs driven by stored representations of familiar objects and scenes. However, the exact mechanisms that subserve such representational processes in the cortex are not yet fully understood. It is known that the entire representational process occurs extremely rapidly and involves a wide cortical network. This network includes both posterior areas of the ventral visual stream, running from striate cortex to inferotemporal cortex, and more anterior areas that lie within the prefrontal cortex (Bullier, 2001; Rainer and Miller, 2000). Within 150200 milliseconds the visual information is propagated through a series of brain regions with bottom-up (posterior) and top-down (prefrontal) influences that lead processing to converge upon an accurate representation of the observed object (Bar, 2003). The feedforward processing in the ventral visual stream itself takes around $100 \mathrm{~ms}$ to reach high-level representational areas in the inferotemporal cortex, and this seems to be sufficient for coarse and rapid categorisation of objects (Liu et al., 2009; VanRullen and Thorpe, 2001). But early feedback from the prefrontal cortex is also considered crucial, since it provides global shape information that subsequently enables posterior cortical areas to 
constrain representational processing to only a few likely interpretations of the observed object (Bar, 2003; Bar et al., 2006). Finally, neural activation in the posterior cortex persists for another couple of hundred milliseconds, lasting around $300 \mathrm{~ms}$ in total. This may be attributable to priming-related shortterm memory processes (as suggested by Bar, 2003) or long-term memory processes (Subramaniam et al., 2000).

The timing of visual object representation is rapid and starts to involve higher-level areas after approx. $100 \mathrm{~ms}$ (Bullier, 2001). Such timing closely coincides with cortical high frequency oscillatory responses, also known as gamma-band activity (GBA; $>30 \mathrm{~Hz})$. GBA arises in neurons of the visual cortex after approx. $100 \mathrm{~ms}$ and has repeatedly been proposed to play an important role in subserving object representation. This review will focus on the accumulated evidence that associates gamma-band oscillations to visual processing. We will argue that the earlier, evoked GBA reflects an initial stage of concurrent bottom-up and top-down processing in vision, while the later, induced GBA is a neural marker of more downstream representational processing which ensures efficient and timely information intake and integration.

\section{Gamma-band activity as a mechanism for the integration of visual information}

Information processing in the brain during perception relies on multiple coding strategies. The strategies employed by a particular neural process will be dependent on the tuning properties of different neurons that subserve it as well as the coding density of neural assemblies to which they belong (Spratling, 2004). A network that is responsible for the perception of objects relies on groups of neurons tuned to different visual properties such as orientation, colour, texture, depth, motion or shape. These neurons are widely distributed, being located in different columns within a single area (orientation columns or colour blobs in V1) or in different areas of the extrastriate cortex altogether (shape in the lateral occipital cortex, motion in middle temporal area, colour in V4/V8). Von der

Malsburg (1990) proposed that temporally synchronous firing would allow coordinated coding of 
different features belonging to the same object within and between these non-contiguous network elements. Synchronised neuronal activity in the gamma-band range was indeed found to relate to the properties of visual stimuli in a series of studies on anesthetised cats, showing enhancements for stimuli that allowed for coherent percepts (Gray and Singer, 1989; for a review see Singer and Gray, 1995). In these studies, synchronised gamma-band activity was elicited by the stimulus but was neither strictly phase- or time-locked to its onset, thus earning the label 'induced' as opposed to 'evoked'. Induced gamma-band oscillations were found to be well-suited to support visual perception, being fast enough to allow neurons to dynamically fall in and out of neural assemblies responsible for integrating the representations of various aspects of everyday scenes (Singer, 1993).

For these same reasons, induced temporal synchrony in the gamma-band is proposed to be the most likely candidate to support a series of other cognitive functions that require rapid and flexible integrative processing in vision, such as awareness, attention and memory. The role of induced GBA in visual cognition has been recently reviewed in great detail by Tallon-Baudry (2009; also see earlier reviews by Kaiser and Lutzenberger, 2003; Keil et al., 2001; Müller et al., 2000; Tallon-Baudry and Betrand, 1999). Evoked GBA, which is both phase and time-locked to stimulus onset, was previously considered to reflect bottom-up processing of image features. However, more recent findings have led to a somewhat altered view on evoked GBA, and it is now perceived as a marker of visual processing that reflects both bottom-up and top-down influences. In this review, the focus is on reports of evoked and induced gamma-band modulations that directly relate to detection, discrimination and classification performance in vision and thus demonstrate that oscillatory synchrony is a neural correlate of visual perception. 


\section{Evoked GBA}

\section{Evoked GBA as a correlate of bottom-up stimulus processing}

In the electroencephalogram (EEG), evoked GBA is among the first responses that follow visual stimulation. Several early studies of brain signals evoked by simple visual stimuli (light flashes, gratings or reversing checkerboards) reported multi-cycled high-frequency oscillations, superimposed on the broad-band visual evoked potential (VEP; Cracco and Cracco, 1978; Sannita et al., 1995; Whittaker and Siegfried, 1983). These so-called "wavelets" - not to be confused with the waveletmethod for spectral analysis - were extracted by high-pass filtering the broad-band signal in the gamma-band range. By contrast, contemporary studies usually extract GBA by applying a timefrequency transform to the event-related potential (ERP) or by computing a measure of phase-locking, such as the phase-locking factor (Tallon-Baudry et al., 1997a). In most studies, evoked GBA peaks at approximately 80-120 ms following stimulus onset. A key characteristic of evoked GBA is strong inter-trial coherence or phase-locking in the frequency range between approximately $30-50 \mathrm{~Hz}$. Its scalp distribution in the EEG is centred over the occipital cortex. Early studies largely excluded a contribution of the electro-retinogram or sub-cortical sources (Lopez and Sannita, 1997; Sannita et al., 1995; Whittaker and Siegfried, 1983). More recent studies with sufficient spatial resolution have identified occipital and occipital-temporal areas as sources of evoked GBA (Gruber et al., 2006; Narici et al., 2003), which matches results of intracranial recordings in humans (Lachaux et al., 2000) and monkeys (Fries et al., 2001; Rols et al., 2001).

Early latency of evoked GBA points to an involvement in sensory stages of visual information processing. Accordingly, several studies have demonstrated that the amplitude of evoked GBA is determined by the properties of the stimulus, including its luminance (Rols et al., 2001), contrast (Sannita et al., 1995; Schadow et al., 2007), size (Busch et al., 2004; Narici et al., 2003), spatial frequency (Bodis-Wollner et al., 2001; Fründ et al., 2007a; Sannita et al., 1995; Tzelepi et al., 2000), or 
location in the visual field (Busch et al., 2004; Lopez and Sannita, 1997; Narici et al., 2003). These findings from human scalp-recorded GBA are in agreement with animal studies using multi-unit activity and local field potentials, which revealed that gamma synchronization is highly dependent on stimulus features such as orientation and direction of movement (Friedman-Hill et al., 2000; Frien et al., 2000; Siegel and Konig, 2003). Together, this suggests that evoked GBA could reflect the activation of feature selective channels in retinotopically organized cortical visual areas which are directly involved in the bottom-up neural representation of a stimulus, similarly to the earliest components of VEPs (cf. Tobimatsu and Celesia, 2006; White, 1995). But unlike the earliest VEP components, evoked GBA is also susceptible to numerous top-down influences. In this review, we will argue that bottom-up stimulus effects and top-down influences are not simply two concurrent effects on the same signal. Rather, we argue that evoked GBA reflects an early processing level where bottom-up stimulus representations interact directly with top-down influences.

\section{Evoked GBA reflects interactions between stimulus representation and task set}

Most reported top-down effects on evoked GBA can be described as effects of task set. Task set refers to a configuration of perceptual, attentional, mnemonic, and motor processes necessary to perform a task ("search for the letter X", "press button A for targets", or "respond as fast as possible"). A critical aspect of task sets, such as the preparation for a difficult task or a specific target stimulus, is that they are formed before the critical stimulus is presented and that they are actively maintained throughout the experiment (cf. Sakai, 2008). Such preparatory effects have been demonstrated by studies of anticipation and task difficulty. These studies revealed that amplitudes of evoked GBA are larger for anticipated stimuli (Fründ et al., 2008b), for speeded compared to slow manual responses (Fründ et al., 2007b) and for difficult compared to easy discrimination tasks (Senkowski and Herrmann, 2002). Evoked GBA was also found to correlate with speed and accuracy of discrimination of a circular arrangement of Gabors embedded in an otherwise random Gabor array; its latency 
correlated with reaction times while its amplitude correlated with accuracy (Schadow et al., 2009). Furthermore, effects of task set have been found in target detection experiments, where the task set implies the storage of a specific target template and subsequent matching of stimuli with this template. Several studies have found that GBA evoked by targets (simple geometric shapes) is larger than GBA evoked by standards (Busch et al., 2006b; Herrmann and Mecklinger, 2000; Kim et al., 2008). According to Engel et al. (2001), attention that is directed towards specific features can synchronize subthreshold oscillations of feature-selective assemblies prior to stimulus presentation. This idea rests on the observation by Fries et al. (2001) that ongoing local field potential fluctuations in the gammaband in cat visual cortex are highly coherent for cells with similar feature preferences but incoherent for cells with different feature preferences. As a consequence of this, other neuronal populations that do not code for the expected feature would not be prepared by means of synchronized subthreshold oscillations and upon stimulus presentation would produce lower evoked responses than the subthreshold-synchronised populations. A critical component of this model is that effects of task set are not enacted via separate, mediating "attention assemblies" but through an attentional modulation of the same neural assemblies that represent the stimulus. Support for this model is provided by Busch et al. (2006b), who found that the magnitude of the target effect on evoked GBA was proportional to the area occupied by the critical target feature and thus to the size of the neural assembly representing the target. This was in contrast to target effects on ERPs, which were independent of the size of the neural assembly representing the stimulus. Together, the current literature suggests that evoked GBA is a neural marker which reflects interactions between bottom-up stimulus representations and top-down influences due to task set.

\section{Is evoked GBA a correlate of object representations?}

Currently, the main controversy in high frequency oscillatory activity research concerns the involvement of evoked GBA in processes that subserve object classification. The "match-and- 
utilization model" by Herrmann et al. (2004b) suggests that evoked GBA is modulated not only when a stimulus matches the expectations induced by the task set but whenever a stimulus matches an existing memory trace. It is assumed that this matching effect does not require a specific stimulus template and thus occurs even when this match is entirely task-irrelevant or when subjects simply discriminate between known and unknown stimuli. This assumption is supported by the finding that evoked GBA is enhanced for images of nameable, meaningful objects compared to unnameable, nonsense objects (Herrmann et al., 2004a; Morup et al., 2006). Furthermore, Fründ et al. (2008a) found that evoked GBA differentiated between meaningful and nonsense objects when participants performed speeded discriminations. There was no effect when responses to the stimuli were delayed. Fründ et al. (2008a) concluded that the early visual processing stages reflected in evoked GBA are affected by object classification only when subjects are pushed to perform rapid stimulus categorization based on coarse and global stimulus features.

However, it should be noted that effects of object classification on evoked GBA have not been consistently found. In fact, the majority of previous studies have failed to find differences in evoked GBA elicited by meaningful and nonsense objects (cf. Busch et al., 2006a; Gruber and Müller, 2005; Gruber et al., 2006; Martinovic et al., 2009; Tallon-Baudry et al., 1997a). In order to clarify the role of evoked GBA in object classification, a recent study by Martinovic et al. (2008b) looked at evoked GBA modulations in a series of experiments that selectively varied the amount of features in object images, for example contrasting black and white line drawings with the same drawings with added greyscale texture or colour. The role of certain image features in the object classification process is reflected by response times, with features such as colour hastening classification (Rossion and Pourtois, 2004) and features such as higher visual complexity slowing it (Ellis and Morrison, 1998). Martinovic et al. (2008b) also presented colour-diagnostic objects either in their typical colours or in an altered, atypical colour (e.g. a purple banana) thus maintaining the amount of features constant but slowing the 
classification process for atypical-colour object images. The aim of the study was to determine whether evoked GBA relates to the image feature processing itself or to the role of those features in the classification process. Martinovic et al. (2008b) found that evoked GBA amplitude always increased when more image features had to be coded, without reflecting the features' qualitative contribution to the classification process. Similar to the sensory-related ERP component P1, evoked GBA was enhanced both for coloured stimuli and for more visually complex line drawings, although they have opposite effects on the speed of object classification. Furthermore, evoked GBA and the P1 component of the ERP did not differ in amplitude between colour-diagnostic objects presented in typical or atypical colours, although atypically-coloured objects were associated with response time costs and modulations of later, representation-related components of the ERP. A possible interpretation of these negative findings is that evoked GBA is not a neural marker of cortical object representation, although it can be affected by "objecthood" in paradigms that rely on a coarse categorisation of stimuli, e.g. discriminating between images of real objects and their scrambled versions. The experiments that contrasted evoked GBA between meaningful and nonsense objects (Busch et al., 2006a; Gruber and Müller, 2005; Herrmann et al., 2004a) relied on different stimulus sets, some coloured, some with linedrawings; they also used various methods to scramble or disassemble the object images and create nonsense objects. This may have resulted in differences between the real and nonsense objects in part structure, connectedness and closure, which are factors that influence perceptual grouping and thus objecthood (for more detail on objecthood, see Scholl, 2001). The influence of such perceptual grouping factors on evoked GBA amplitude could explain why object-selectivity effects have not been consistently found.

\section{Induced GBA}

Induced GBA as a correlate of stimulus detection, discrimination and classification 
Induced gamma-band activity in scalp-recorded EEG data usually appears as bursts of high frequency (approximately 30-80 Hz) oscillatory activity in the time range between 200-400 ms (Busch et al., 2006a; Gruber et al., 2004; Tallon-Baudry et al., 1997a). Induced GBA measured with MEG or depth-EEG is usually longer lasting and extends to higher frequencies (Hoogenboom et al., 2006; Lachaux et al., 2005; Siegel et al., 2008; Wyart and Tallon-Baudry, 2008). These bursts are not phaselocked to stimulus onset, so averaging epochs of raw data across trials would cancel out the response. To analyze induced GBA, a time-frequency transform has to be applied to the single trials before averaging (see Tallon-Baudry and Betrand, 1999). Induced gamma-band oscillations can be observed in early visual areas soon after a visual stimulus is presented. In anesthetised monkeys, within-area gamma-band synchrony in V1 peaks around $100 \mathrm{~ms}$ after a simple flashing stimulus is presented, while in V4 this occurs approximately $40 \mathrm{~ms}$ later with a decreased response magnitude (Rols et al., 2001). Lachaux et al. (2005) used depth EEG to investigate large-scale cortical dynamics during the perception of upright or inverted Mooney faces in four epileptic patients. In depth EEG, intracerebral electrodes are used to directly record the activity from brain structures potentially involved in the generation of epileptic activity and Lachaux et al. (2005) selected a group of patients with electrodes positioned in various posterior cortical areas. This permitted them to identify the time-course of induced GBA in different areas of the visual cortex. They found that correctly identified faces induced increases in gamma-band activity in a distributed network, with an early burst in the fusiform gyrus and the adjacent lateral occipital cortex $(<200 \mathrm{~ms})$ which was immediately followed by a posterior-anterior propagation of activity and a deactivation in the primary visual cortex. It was suggested that betweenarea synchrony emerges even later, around $250 \mathrm{~ms}$, and reflects a coming together of bottom-up and top-down processes in widely distributed areas that leads to successful object classification. Such a process was thought to be reflected by a transient burst of induced GBA recorded from the scalp with EEG, which usually arises between 200 and 400 ms (Tallon-Baudry, 2003; Tallon-Baudry and Betrand, 1999; although this has recently been challenged by the findings of Yuval-Greenberg et al., 2008). 
Early studies in the field focused mostly on the relation of induced GBA to various stimulus attributes (in particular stimulus coherence), but some recent studies have attempted to demonstrate its more direct involvement in perception by investigating correlations between induced GBA amplitude and detection latencies. For instance, Womelsdorf et al. (2006) found correlations between the power of induced gamma-band activity pre- and post-stimulus and the speed of detection of attended as opposed to unattended visual stimuli in area V4 of awake monkeys. An EEG study by Koch et al. (2009) investigated induced GBA whilst participants detected changes in the velocity of concentrically moving gratings whose contrast was parametrically modulated. The Koch et al. (2009) study demonstrated that the amplitude of induced GBA was not only systematically related to task-irrelevant stimulus contrast but also predicted the latency of behavioural responses to task-relevant velocity changes. Furthermore, Koch et al. (2009) also observed induced GBA that was sustained through the entire stimulation period, similar to MEG findings on induced GBA in motion processing (Hoogenboom et al., 2006; Swettenham et al., 2009). One of the earliest studies on gamma-band in the human EEG during observation of coherently or incoherently moving bars also found sustained induced GBA enhancements for coherent motion (Müller et al., 1997).

Another major line of research into induced GBA has focused on its role in the formation of a coherent percept and in object representation. A multitude of studies has demonstrated that induced GBA amplitude is enhanced for stimuli that constitute a coherent percept (Gruber et al., 2002; Gruber and Müller, 2005; Keil et al., 1999; Rodriguez et al., 1999; Tallon-Baudry et al., 1996, 1997a; TallonBaudry et al., 1997b). In particular, a transient, broadband burst of induced gamma-band activity is often observed in studies that require classification of foveally presented objects. The amplitude of such induced GBA is attenuated for repeated presentations of the same meaningful object (Gruber et al., 2004; Gruber and Müller, 2002) leading to suggestions that it could be a neural correlate of a relatively late stream of representational processing connected to visual memory processes. This 
relatively short, induced GBA burst observed in object classification paradigms does not seem to be a mere fixed-latency epiphenomenon of visual perception. Martinovic et al. (2008a; 2007) have related the peak latency of the induced GBA to the time point of object classification. In these studies, participants covertly named two-dimensional line drawings of objects that are characterised by a predominant environmental orientation (chair, house or cow, for example). The objects were presented either in their everyday orientation or misoriented in the picture plane $\left( \pm 60^{\circ}\right.$ or $\left.\pm 120^{\circ}\right)$. Classification of such misoriented images is delayed due to the necessity to employ view-dependent recognition processes while accessing the name of the object (Jolicoeur et al., 1998; Lawson and Jolicoeur, 1998, 2003). Martinovic et al. (2008a; 2007) found that the delay in induced GBA's peak latency corresponded to delays in response times for misoriented objects, connecting the time-course of induced GBA to the timing of representational processing.

\section{Induced GBA - fact or artifact?}

Extensive research on induced high frequency oscillations in animals and humans has convincingly related this response to representational processes that occur during visual cognition. However, a recent study by Yuval-Greenberg et al. (2008) has questioned the cortical origin of induced GBA in EEG by demonstrating that the contraction of ocular muscles during miniature eye movements can cause high frequency artifacts in EEG. The dynamics of these miniature saccades are similar to induced GBA, peaking at around 200-350 ms. Yuval-Greenberg et al. (2009) have subsequently called for a full reevaluation of EEG research into induced GBA that reported transient bursts of broadband activity. It is indeed essential to determine whether a scalp-recorded response is influenced by a) effects of microsaccades on genuine visual processing or b) transient oculomotor potentials occurring in the high frequency range of the EEG. Microsaccades play an important role in vision and are well-known to be affected by both sensory and cognitive factors (for recent reviews see Martinez-Conde et al., 2009; Rolfs, 2009). These miniature fixational eye movements play a very complex role in perception, 
modulating neuronal firing rates in both striate (V1) and extrastriate cortex (V2, V4) within approx. 100-120 ms of the execution of the eye movement (Leopold and Logothetis, 1998). In YuvalGreenberg et al. (2008), the observed connection between microsaccades and induced GBA in the EEG was correlational - microsaccades coincided with GBA, indicating an artifactual origin. But microsaccades and induced gamma band activity could also correlate because they are triggered by the same perceptual processes. In line with this idea, Bosman et al. (2009) studied gamma-band activity in areas V1 and V4 of awake monkeys and found that microsaccadic patterns affected synchronisation in the gamma-band range, which in turn predicted the response latency variability. Moreover, onsets of microsaccade influenced activity in the visual cortex correspond tightly to the timing of the earliest bursts of induced high frequency oscillations, with microsaccadic activity peaking at approx. 90-120 ms in V1 to V4 (Leopold and Logothetis, 1998) and induced gamma-band activity peaking at around $100 \mathrm{~ms}$ in V1 and $140 \mathrm{~ms}$ in V4 (Rols et al., 2001).

Other studies also suggest that there is more to induced GBA in EEG than being a mere artifact of saccadic spike potentials. Several recent studies reveal transient increases in activity during visual working memory or face classification paradigms which cannot be explained as occular artifacts since the topographic EEG maps do not exhibit artifact-associated increases at frontal sites (Haenschel et al., 2009; Uhlhaas et al., 2009). Furthermore, accounts of induced GBA as an artifact or as a genuine reflection of cortical activity are not necessarily mutually exclusive. Both microsaccadic patterns and brain activity can be influenced by common cognitive factors, and both occur in a similar time window. Thus, it is possible that a large-amplitude artifactual response in the EEG could obscure a much smaller, cortically generated oscillation. Therefore, it is essential to develop tools for artifact removal that will permit the exclusion of signals induced by ocular muscle activity during microsaccades (Keren et al., 2010). Independent component analysis (ICA) methods are currently under development and initial results look extremely promising - after removing transient spike potentials that accompany 
miniature eye movements, an ongoing narrowband $(50-70 \mathrm{~Hz})$ oscillatory response can be revealed (Hassler and Gruber, 2009). This indicates that it is possible to obtain a high frequency signal from the cortex by using scalp EEG and indicates a positive outlook for the future of non-invasive human research into mechanisms of cortical object representation. Further studies are needed to fully specify the relation of microsaccades and oscillatory synchrony (see Melloni et al., 2009). Once reliable tools for removal of occular artifacts have been developed, some studies on object representation and induced GBA (e.g. Martinovic et al., 2008a; Martinovic et al., 2007, which focused on the transient peak itself) will need to be re-examined to assess if the observed transient GBA bursts reflected a modulation of eye movements, modulation of high frequency oscillatory synchrony, or both.

\section{Future directions}

Relations between microsaccades and induced high frequency oscillatory activity are currently one of the most important research topics. When humans freely observe complex visual scenes, salient features such as human faces attract most fixations, and microsaccadic rates are highest whilst foveating such locations (Otero-Millan et al., 2008). Both evoked and induced GBA are also highest for foveally presented stimuli (Busch et al., 2004; Swettenham et al., 2009). Furthermore, induced GBA amplitude is modulated by the amount of perceptual resources that can be allocated to the foveallypresented unattended objects (Martinovic et al., 2009). The role of gamma-band oscillations in attentive as opposed to inattentive processing both peripherally and foveally needs to be explored further, as the strategic functions of visual attention are likely to vary retinotopically (Handy and Khoe, 2005). While there may be a tight relation between microsaccades and induced GBA for stimuli presented foveally, a different pattern may emerge for peripherally presented attended or unattended stimuli. It is also necessary to explore the dynamics of gamma-band oscillations in high-level visual, temporal and prefrontal areas during complex activities such as reading (Jung et al., 2008). Measures of directed cortical information flow between sources of gamma-band activity (Supp et al., 2007) can also reveal 
much about the particular ways in which different brain regions interact within the perceptual and attentional networks. These measures could be particularly useful in determining the dynamics of occasionally reported induced GBA decreases relative to baseline - Lachaux et al. (2005) observed that concurrently with the activation of higher-level visual areas, V1 gamma synchrony decreases and Jung et al. (2008) observed decreases in ventral lateral prefrontal cortex for attended words concurrently with persistent enhancements in posterior areas. The role of these desynchronisations is not fully understood but it is assumed that they are likely to reflect the supression of processing in task-irrelevant brain regions. Finally, the properties of miniature eye movement artifacts that can affect induced GBA in EEG recordings need to be fully specified, and methods of artifact removal have to be perfected (Keren et al., 2010). The possibility that object classification can be reflected in early, involuntary eye movements is in itself very intriguing and worthy of further research.

Schroeder and Lakatos (2009) proposed that attentional selection is achieved by utilising the regularities of the visual environment as well as regularities in exploratory motor and eye movement patterns in order to enforce oscillatory entrainment to a task-relevant input stream. They propose that in the absence of a low frequency rhythm that oscillations can entrain to, attention is characterised by an extended increase in gamma-band synchrony. It would be promising to investigate gamma-band activity in paradigms that require ongoing visually-guided behaviour or ongoing observation of dynamic, complex visual scenes in order to examine patterns of oscillatory synchrony in multiple frequency bands that relate to both the processing of the visual stimulus and of the required response. Ongoing gamma-band activity has already been demonstrated during the 5 -second observation of rotating ambiguous figures (Keil et al., 1999).

With regards to evoked GBA, the main open question concerns the early objecthood effects which are not consistently reported but do seem to occur with certain stimulus sets and tasks. By selecting a set of images of real, meaningful objects and then parametrically modulating the properties of the 
nonsense, scrambled versions of these objects it could be possible to ascertain which attributes are crucial in driving the modulations of evoked GBA amplitude. This could indicate whether the effects of coarse classification depend on certain uncontrolled low-level image attributes (e.g. visual complexity, aspect ratio) or if they are due to the closed or open line structure of the stimuli, which can be indicative of objecthood.

\section{Concluding comments}

Vision must rely on mechanisms that allow for flexible and dynamic coding between distributed neural assemblies. The nature of these mechanisms presents a fundamental question for visual neuroscience. High frequency oscillatory synchrony is a likely candidate for one such mechanism since it allows neurons to rapidly fall in and out of assemblies that code for various aspects of visual scenes, coordinating dispersed activity elicited by a single object or event. This role of GBA in neuronal communication is, of course, not limited to visual processing, and is likely to be essential to cortical computation itself.

Although high frequency oscillatory synchrony is most likely a mechanism of broad significance for efficient neuronal communication, the attributes of gamma-band synchrony in vision strongly suggest that it is not simply a non-specific marker of cognitive effort: it is established between neurons and neuronal assemblies in the visual cortex both before and after the onset of task relevant events and exhibits complex patterns of propagation through ventral and dorsal visual streams during the representation of meaningful, coherent objects. Correlations between gamma-band synchrony and behaviour further demonstrate their genuine role in representational processes occurring in vision. These findings strongly support the assumption that evoked gamma-band activity reflects an interface of bottom-up and top-down visual processing, while induced high frequency synchrony plays a crucial role in subserving efficient information intake and processing during attentive vision. 


\section{Acknowledgments}

The authors would like to thank Corinna Haenschel for helpful comments on previous versions of the manuscript and Matt Craddock for proofreading one of the final versions of the manuscript.

\section{References}

Bar, M., 2003. A cortical mechanism for triggering top-down facilitation in visual object recognition. J. Cogn. Neurosci. 15, 600-609.

Bar, M., Kassam, K.S., Ghuman, A.S., Boshyan, J., Schmid, A.M., Dale, A.M., Hämäläinen, M.S., Marinkovic, K., Schacter, D.L., Rosen, B.R., Halgren, E., 2006. Top-down facilitation of visual recognition. Proceedings of the National Academy of Sciences of USA 103, 449-454.

Bodis-Wollner, I., Davis, J., Tzelepi, A., Bezerianos, T., 2001. Wavelet transform of the EEG reveals differences in low and high gamma responses to elementary visual stimuli. Clin. Electroencephalogr. 32, 139-144.

Bosman, C.A., Womelsdorf, T., Desimone, R., Fries, P., 2009. A Microsaccadic Rhythm Modulates Gamma-Band Synchronization and Behavior. J. Neurosci. 29, 9471-9480.

Bullier, J., 2001. Integrated model of visual processing. Brain Research Reviews 36, 96-107.

Busch, N.A., Debener, S., Kranczioch, C., Engel, A.K., Herrmann, C.S., 2004. Size matters: effects of stimulus size, duration and eccentricity on the visual gamma-band response. Clin. Neurophysiol. 115, 1810-1820.

Busch, N.A., Herrmann, C.S., Müller, M.M., Lenz, D., Gruber, T., 2006a. A cross-laboratory study of eventrelated gamma band activity in a standard object recognition paradigm. Neuroimage 33, 1169-1177.

Busch, N.A., Schadow, J., Fruend, I., Herrmann, C.S., 2006b. Time-frequency analysis of target detection reveals an early interface between bottom-up and top-down processes in the gamma-band. Neuroimage 29, 11061116.

Cracco, R.Q., Cracco, J.B., 1978. Visual evoked-potential in man: Early oscillatory potentials.

Electroencephalogr. Clin. Neurophysiol. 45, 731-739.

Ellis, A.W., Morrison, C.M., 1998. Real age-of-acquisition effects in lexical retrieval. Journal of Experimental Psychology: Learning, Memory and Cognition 24, 515-523.

Engel, A.K., Fries, P., Singer, W., 2001. Dynamic predictions: oscillations and synchrony in top-down processing. Nature Reviews Neuroscience 2, 704-716.

Friedman-Hill, S., Maldonado, P.E., Gray, C.M., 2000. Dynamics of striate cortical activity in the alert macaque: I. Incidence and stimulus-dependence of gamma-band neuronal oscillations. Cereb. Cortex 10, 1105-1116. Frien, A., Eckhorn, R., Bauer, R., Woelbern, T., Gabriel, A., 2000. Fast oscillations display sharper orientation tuning than slower components of the same recordings in striate cortex of the awake monkey. Eur. J. Neurosci. $12,1453-1465$.

Fries, P., Neuenschwander, S., Engel, A.K., Goebel, R., Singer, W., 2001. Rapid feature selective neuronal synchronization through correlated latency shifting. Nat. Neurosci. 4, 194-200.

Fründ, I., Busch, N.A., Korner, U., Schadow, J., Herrmann, C.S., 2007a. EEG oscillations in the gamma and alpha range respond differently to spatial frequency. Vision Reasearch 47, 2086-2098.

Fründ, I., Busch, N.A., Schadow, J., Gruber, T., Körner, U., Herrmann, C.S., 2008a. Time pressure modulates electrophysiological correlates of early visual processing. PLOS Biology 3, e1675. 
Fründ, I., Busch, N.A., Schadow, J., Korner, U., Herrmann, C.S., 2007b. From perception to action: phase-locked gamma oscillations correlate with reaction times in a speeded response task. BMC Neuroscience 8.

Fründ, I., Schadow, J., Busch, N.A., Naue, N., Korner, U., Herrmann, C.S., 2008b. Anticipation of natural stimuli modulates EEG dynamics: physiology and simulation. Cogn. Neurodynamics 2, 89-100.

Gray, C.M., Singer, W., 1989. Stimulus-specific neuronal oscillations in orientation columns of cat visual cortex. Proceedings of the National Acadamy of Science USA 86, 1698-1702.

Gruber, T., Keil, A., Müller, M.M., 2002. Modulation of human induced gamma band response in a perceptual learning task. J. Cogn. Neurosci. 14.

Gruber, T., Malinowski, P., Müller, M.M., 2004. Modulation of oscillatory brain activity and evoked potentials in a repetition priming task in the human EEG. Eur. J. Neurosci. 19, 1073-1082.

Gruber, T., Müller, M.M., 2002. Effects of picture repetition on induced gamma band responses, evoked

potentials, and phase synchrony in the human EEG. Cognitive Brain Research 13, 377-392.

Gruber, T., Müller, M.M., 2005. Oscillatory brain activity dissociates between associative stimulus content in a repetition priming task in the human EEG. Cereb. Cortex 15, 109-116.

Gruber, T., Trujillo-Barreto, J.N., Giabbiconi, C.M., Valdes-Sosa, P.A., Müller, M.M., 2006. Brain electrical tomography (BET) analysis of induced gamma band responses during a simple object recognition task. Neuroimage 29, 888-900.

Haenschel, C., Bittner, R.A., Waltz, J., Haertling, F., Wibral, M., Singer, W., Linden, D.E.J., Rodriguez, E., 2009.

Cortical Oscillatory Activity Is Critical for Working Memory as Revealed by Deficits in Early-Onset Schizophrenia. J. Neurosci. 29, 9481-9489.

Handy, T.C., Khoe, W., 2005. Attention and sensory gain control: A peripheral visual process? J. Cogn. Neurosci. 17, 1936-1949.

Hassler, U., Gruber, T., 2009. Induced gamma band responses: neuronal origin or eye movement artifact?

Psychophysiology 46, S61-S61.

Herrmann, C.S., Lenz, D., Junge, S., Busch, N.A., Maess, B., 2004a. Memory-matches evoke human gammaresponses. BMC Neuroscience 5, 13.

Herrmann, C.S., Mecklinger, A., 2000. Magnetoencephalographic responses to illusory figures: early evoked gamma is affected by processing of stimulus features. Int. J. Psychophysiol. 38, 265-281.

Herrmann, C.S., Munk, M.H.J., Engel, A.K., 2004b. Cognitive functions of gamma-band activity: memory match and utilization. Trends in Cognitive Sciences 8, 347-355.

Hoogenboom, N., Schoffelen, J.M., Oostenveld, R., Parkes, L.M., Fries, P., 2006. Localizing human visual gamma-band activity in frequency, time and space. Neuroimage 29, 764-773.

Jolicoeur, P., Corballis, M.C., Lawson, R., 1998. The influence of perceived rotary motion on the recognition of rotated objects. Psychonomic Bulletin \& Review 5, 140-146.

Jung, J., Mainy, N., Kahane, P., Minotti, L., Hoffmann, D., Bertrand, O., Lachaux, J.P., 2008. The neural bases of attentive reading. Human brain mapping 29, 1193-1206.

Kaiser, J., Lutzenberger, W., 2003. Induced gamma-band activity and human brain function. The Neuroscientist 9, 475-484.

Keil, A., Gruber, T., Müller, M.M., 2001. Functional correlates of macroscopic high-frequency brain activity in the human visual system. Neuroscience and Biobehavioural Reviews 6, 527-534.

Keil, A., Müller, M.M., Ray, W.J., Elbert, T., Gruber, T., 1999. Human gamma band activity and perception of a gestalt. The Journal of Neuroscience 19, 7152-7161.

Keren, A.S., Yuval-Greenberg, S., Deouell, L.Y., 2010. Saccadic spike potentials in gamma-band EEG:

Characterization, detection and suppression. Neuroimage 49, 2248-2263.

Kim, K.H., Yoon, J., Kim, J.H., Jung, K.Y., 2008. Changes in gamma-band power and phase synchronization with the difficulty of a visual oddball task. Brain Res. 1236, 105-112.

Koch, S.P., Werner, P., Steinbrink, J., Fries, P., Obrig, H., 2009. Stimulus-Induced and State-Dependent

Sustained Gamma Activity Is Tightly Coupled to the Hemodynamic Response in Humans. J. Neurosci. 29, 1396213970. 
Lachaux, J.-P., George, N., Tallon-Baudry, C., Martinerie, J., Hugueville, L., Minotti, L., Kahane, P., Renault, B., 2005. The many faces of the gamma band response to complex visual stimuli. Neuroimage 25, 491-501. Lachaux, J.P., Rodriguez, E., Martinerie, J., Adam, C., Hasboun, D., Varela, F.J., 2000. A quantitative study of gamma-band activity in human intracranial recordings triggered by visual stimuli. Eur. J. Neurosci. 12, 26082622.

Lawson, R., Jolicoeur, P., 1998. The effects of plane rotation on the recognition of brief masked pictures of familiar objects. Memory \& Cognition 26, 791-803.

Lawson, R., Jolicoeur, P., 2003. Recognition thresholds for plane-rotated pictures of familiar objects. Acta Psychologica 112, 17-41.

Leopold, D.A., Logothetis, N.K., 1998. Microsaccades differentially modulate neural activity in the striate and extrastriate visual cortex. Experimental Brain Research 123, 341-345.

Liu, H.S., Agam, Y., Madsen, J.R., Kreiman, G., 2009. Timing, Timing, Timing: Fast Decoding of Object Information from Intracranial Field Potentials in Human Visual Cortex. Neuron 62, 281-290.

Lopez, L., Sannita, W.G., 1997. Magnetically recorded oscillatory responses to luminance stimulation in man. Evoked Potential.-Electroencephalogr. Clin. Neurophysiol. 104, 91-95.

Malsburg, C.v.d., 1990. A neural architecture for the representation of scenes, in: McGaugh, J.L., Weinberger, N.M., Lynch, G. (Eds.), Brain organization and memory: Cells, systems and circuits. Oxford University Press, New York, pp. 356-372.

Martinez-Conde, S., Macknik, S.L., Troncoso, X.G., Hubel, D.H., 2009. Microsaccades: a neurophysiological analysis. Trends in Neurosciences 32, 463-475.

Martinovic, J., Gruber, T., Hantsch, A., Müller, M.M., 2008a. Induced gamma-band activity is related to the time point of object identification. Brain Res. 1198, 93-106.

Martinovic, J., Gruber, T., Müller, M., 2008b. Coding of visual object features and feature conjunctions in the human brain. PLOS ONE 3.

Martinovic, J., Gruber, T., Müller, M.M., 2007. Induced gamma-band responses predict recognition delays during object identification. J. Cogn. Neurosci. 19, 921-934.

Martinovic, J., Gruber, T., Ohla, K., Muller, M.M., 2009. Induced gamma-band activity elicited by visual representation of unattended objects. J. Cogn. Neurosci. 21, 42-57.

Melloni, L., Schwiedrzik, C.M., Rodriguez, E., Singer, W., 2009. (Micro)Saccades, corollary activity and cortical oscillations. Trends in Cognitive Sciences 13, 239-245.

Morup, M., Hansen, L.K., Herrmann, C.S., Parnas, J., Arnfred, S.M., 2006. Parallel factor analysis as an exploratory tool for wavelet transformed event-related EEG. Neuroimage 29, 938-947.

Müller, M.M., Gruber, T., Keil, A., 2000. Modulation of induced gamma band activity in the human EEG by attention and visual information processing. Int. J. Psychophysiol. 38, 283-300.

Müller, M.M., Junghofer, M., Elbert, T., Rochstroh, B., 1997. Visually induced gamma-band responses to coherent and incoherent motion: a replication study. NeuroReport 8, 2575-2579.

Narici, L., Carozzo, S., Lopez, L., Ogliastro, C., Sannita, W.G., 2003. Phase-locked oscillatory similar to 15-to 30$\mathrm{Hz}$ response to transient visual contrast stimulation: neuromagnetic evidence for cortical origin in humans. Neuroimage 19, 950-958.

Otero-Millan, J., Troncoso, X.G., Macknik, S.L., Serrano-Pedraza, I., Martinez-Conde, S., 2008. Saccades and microsaccades during visual fixation, exploration, and search: Foundations for a common saccadic generator. Journal of Vision 8.

Palmer, S.E., 1999. Vision Science: Photons to phenomenology. MIT Press, Cambridge, MA.

Rainer, G., Miller, E.K., 2000. Effects of visual experience on the representation of objects in the prefrontal cortex. Neuron 27, 179-189.

Rodriguez, E., George, N., Lachaux, J.-P., Martinerie, J., Renault, B., Varela, F.J., 1999. Perception's shadow: long-distance synchronization of human brain activity. Nature 397, 430-433.

Rolfs, M., 2009. Microsaccades: Small steps on a long way. Vision Res. 49, 2415-2441.

Rols, G., Tallon-Baudry, C., Girard, P., Bertrand, O., Bullier, J., 2001. Cortical mapping of gamma oscillations in areas V1 and V4 of the macaque monkey. Visual Neuroscience 18, 527-540. 
Rossion, B., Pourtois, G., 2004. Revisiting Snodgrass and Vanderwart's object pictorial set: The role of surface detail in basic-level object recognition. Perception 33, 217-236.

Sakai, K., 2008. Task set and prefrontal cortex. Annu. Rev. Neurosci. 31, 219-245.

Sannita, W.G., Lopez, L., Piras, C., Dibon, G., 1995. Scalp-recorded oscillatory potentials-evoked by transient pattern-reversal visual stimulation in man. Evoked Potential.-Electroencephalogr. Clin. Neurophysiol. 96, 206218.

Schadow, J., Dettler, N., Paramei, G.V., Lenz, D., Frund, I., Sabel, B.A., Herrmann, C.S., 2009. Impairments of Gestalt perception in the intact hemifield of hemianopic patients are reflected in gamma-band EEG activity. Neuropsychologia 47, 556-568.

Schadow, J., Lenz, D., Thaerig, S., Busch, N.A., Frund, I., Rieger, J.W., Herrmann, C.S., 2007. Stimulus intensity affects early sensory processing: Visual contrast modulates evoked gamma-band activity in human EEG. Int. J. Psychophysiol. 66, 28-36.

Scholl, B.J., 2001. Objects and attention: the state of the art. Cognition 80, 1-46.

Schroeder, C.E., Lakatos, P., 2009. Low-frequency neuronal oscillations as instruments of sensory selection. Trends in Neurosciences 32, 9-18.

Senkowski, D., Herrmann, C.S., 2002. Effects of task difficulty on evoked gamma activity and ERPs in a visual discrimination task. Clin. Neurophysiol. 113, 1742-1753.

Siegel, M., Donner, T.H., Oostenveld, R., Fries, P., Engel, A.K., 2008. Neuronal Synchronization along the Dorsal Visual Pathway Reflects the Focus of Spatial Attention. Neuron 60, 709-719.

Siegel, M., Konig, P., 2003. A functional gamma-band defined by stimulus-dependent synchronization in area 18 of awake behaving cats. J. Neurosci. 23, 4251-4260.

Singer, W., 1993. Synchronization of cortical activity and its putative role in information-processing and learning. Annu. Rev. Physiol. 55, 349-374.

Singer, W., Gray, C.M., 1995. Visual feature integration and the temporal correlation hypothesis. Annu. Rev. Neurosci. 18, 555-586.

Spratling, M.W., 2004. Local versus distributed: A poor taxonomy of neural coding strategies. Behavioral and Brain Sciences 27, 700-702.

Subramaniam, S., Biederman, I., Madigan, S., 2000. Accurate identification but no priming and chance recognition memory for pictures in RSVP sequences. Visual Cognition 7, 511-535.

Supp, G.G., Schlogl, A., Trujillo-Barreto, N., Muller, M.M., Gruber, T., 2007. Directed cortical information flow during human object recognition: analyzing induced EEG gamma-band responses in brain's source space. PLoS ONE 2, e684.

Swettenham, J.B., Muthukumaraswamy, S.D., Singh, K.D., 2009. Spectral Properties of Induced and Evoked Gamma Oscillations in Human Early Visual Cortex to Moving and Stationary Stimuli. J. Neurophysiol. 102, 12411253.

Tallon-Baudry, C., 2003. Oscillatory synchrony and human visual cognition. J. Physiol.-Paris 97, 355-363. Tallon-Baudry, C., 2009. The roles of gamma-band oscillatory synchrony in human visual cognition. Front. Biosci. 14, 321-332.

Tallon-Baudry, C., Bertrand, O., Delpuech, C., Pernier, J., 1996. Stimulus specifity of phase-locked and nonphase-locked $40 \mathrm{~Hz}$ visual response in human. Eur. J. Neurosci. 16, 4240-4249.

Tallon-Baudry, C., Bertrand, O., Delpuech, C., Pernier, J., 1997a. Oscillatory gamma-band $(30-70 \mathrm{~Hz})$ activity induced by a visual search task in humans. J. Neurosci. 17, 722-734.

Tallon-Baudry, C., Bertrand, O., Wienbruch, C., Ross, B., Pantev, C., 1997b. Combined EEG and MEG recordings of visual 40-Hz responses to illusory triangles in human. NeuroReport 8, 1103-1107.

Tallon-Baudry, C., Betrand, O., 1999. Oscillatory gamma activity in humans and its role in object representation. Trends in Cognitive Sciences 3, 151-162.

Tobimatsu, S., Celesia, G.G., 2006. Studies of human visual pathophysiology with visual evoked potentials. Clin. Neurophysiol. 117, 1414-1433.

Tzelepi, A., Bezerians, T., Bodis-Wollner, I., 2000. Functional properties of sub-bands of oscillatory brain waves to pattern visual stimulation in man. Clin. Neurophysiol. 111, 259-269. 
Uhlhaas, P.J., Roux, F., Singer, W., Haenschel, C., Sireteanu, R., Rodriguez, E., 2009. The development of neural synchrony reflects late maturation and restructuring of functional networks in humans. Proc. Natl. Acad. Sci. U. S. A. $106,9866-9871$.

Ullman, S., 2007. Object recognition and segmentation by a fragment-based hierarchy. Trends in Cognitive Sciences 11, 58-64.

VanRullen, R., Thorpe, S.J., 2001. The time course of visual processing: from early perception to decisionmaking. J. Cogn. Neurosci. 13, 454-461.

White, C.T., 1995. REFLECTIONS ON VISUAL-EVOKED CORTICAL POTENTIALS AND SELECTIVE ATTENTION METHODOLOGICAL AND HISTORICAL. International Journal of Neuroscience 80, 13-30.

Whittaker, S.G., Siegfried, J.B., 1983. Origin of wavelets in the visual evoked-potential. Electroencephalogr. Clin. Neurophysiol. 55, 91-101.

Womelsdorf, T., Fries, P., Mitra, P.P., Desimone, R., 2006. Gamma-band synchronization in visual cortex predicts speed of change detection. Nature 439, 733-736.

Wyart, V., Tallon-Baudry, C., 2008. Neural dissociation between visual awareness and spatial attention. J. Neurosci. 28, 2667-2679.

Yuval-Greenberg, S., Deouell, L.Y., 2009. The Broadband-Transient Induced Gamma-Band Response in Scalp EEG Reflects the Execution of Saccades. Brain Topography 22, 3-6.

Yuval-Greenberg, S., Tomer, O., Keren, A.S., Nelken, I., Deouell, L.Y., 2008. Transient induced gamma-band response in EEG as a manifestation of miniature saccades. Neuron 58, 429-411. 